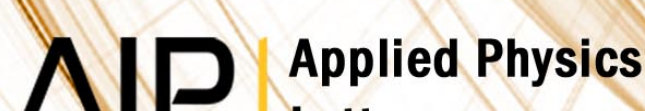 \\ Letters
}

\section{Magnetic field effects on the electroluminescence of organic light emitting devices: A tool to indicate the carrier mobility}

B. F. Ding, Y. Yao, Z. Y. Sun, C. Q. Wu, X. D. Gao et al.

Citation: Appl. Phys. Lett. 97, 163302 (2010); doi: 10.1063/1.3505343

View online: http://dx.doi.org/10.1063/1.3505343

View Table of Contents: http://apl.aip.org/resource/1/APPLAB/v97/i16

Published by the American Institute of Physics.

\section{Related Articles}

Control of coherent acoustic phonon generation with external bias in InGaN/GaN multiple quantum wells Appl. Phys. Lett. 100, 101105 (2012)

Small molecular phosphorescent organic light-emitting diodes using a spin-coated hole blocking layer Appl. Phys. Lett. 100, 083304 (2012)

Small molecular phosphorescent organic light-emitting diodes using a spin-coated hole blocking layer APL: Org. Electron. Photonics 5, 53 (2012)

AIGaN-based ultraviolet light-emitting diodes using fluorine-doped indium tin oxide electrodes Appl. Phys. Lett. 100, 081110 (2012)

Temperature dependent efficiency droop in GalnN light-emitting diodes with different current densities Appl. Phys. Lett. 100, 081106 (2012)

\section{Additional information on Appl. Phys. Lett.}

Journal Homepage: http://apl.aip.org/

Journal Information: http://apl.aip.org/about/about_the_journal

Top downloads: http://apl.aip.org/features/most_downloaded

Information for Authors: http://apl.aip.org/authors

\section{ADVERTISEMENT}

\section{NEW!}

iPeerReview AlP's Newest App

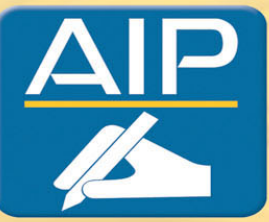

Authors...

Reviewers...

Click the status of

submitted papers remotely!

ADPublishing 


\title{
Magnetic field effects on the electroluminescence of organic light emitting devices: A tool to indicate the carrier mobility
}

\author{
B. F. Ding, ${ }^{1,2}$ Y. Yao, ${ }^{2}$ Z. Y. Sun, ${ }^{2}$ C. Q. Wu, ${ }^{2}$ X. D. Gao, ${ }^{2}$ Z. J. Wang,${ }^{2}$ X. M. Ding, ${ }^{2}$ \\ W. C. H. Choy, ${ }^{1, a)}$ and X. Y. Hou ${ }^{2}$ \\ ${ }^{1}$ Department of Electrical and Electronic Engineering, The University of Hong Kong, Pokfulam Road, \\ Hong Kong \\ ${ }^{2}$ State Key Laboratory of Surface Physics, Fudan University, Shanghai 200433, People's Republic of China
}

(Received 9 September 2010; accepted 3 October 2010; published online 21 October 2010)

\begin{abstract}
The magnetoelectroluminescence (MEL) of organic light emitting devices with a $\mathrm{N}, \mathrm{N}^{\prime}$ bis(1-naphthyl)-N, $\mathrm{N}^{\prime}$-diphenyl-1,1'-biphentl-4,4'-diamine:tris-(8-hydroxyquinoline) aluminum $\left(\mathrm{NPB}: \mathrm{Alq}_{3}\right)$ mixed emission layer (EML) has been investigated. We find that MEL is maximized when the volume ratio of NPB of the mixed EML reaches $30 \%$ and the EML thickness is $40 \mathrm{~nm}$. The features of MEL under various magnetic field strengths are insensitive to the change in EML thickness and mixing ratio. Meanwhile, MEL has a close relationship with the carrier mobility. We have conducted a theoretical study to further verify the relationship. Our experimental and theoretical results confirm that MEL can function as a tool to indicate the mobility. (C) 2010 American Institute of Physics. [doi:10.1063/1.3505343]
\end{abstract}

Magnetic field can moderately modify the electroluminescence (EL) of organic light emitting devices (OLEDs), namely, organic magnetoelectroluminescence (MEL). ${ }^{1-5}$ MEL is quantitatively expressed with $\Delta \mathrm{EL} / \mathrm{EL}=[(\mathrm{EL}(\mathrm{B})$ $-\operatorname{EL}(0)] / \operatorname{EL}(0)$, where $\operatorname{EL}(B)$ and $\operatorname{EL}(0)$ are the EL intensity with and without external magnetic field $B$, respectively. ${ }^{6}$ MEL provides a reliable way to investigate the spin related exciton dynamics, ${ }^{6-10}$ the mechanism of the carrier-to-photon conversion in organic semiconductors, ${ }^{8-12}$ and even the operation mechanism of magnetic sensor and avian magnetic compass. ${ }^{13}$ Some methods have been used to control and tune MEL, such as changing applied voltage $e^{4,5,12,14}$ and inserting insulating layer of $\mathrm{LiF}^{5}$ However, it is still not clear which factor governs MEL. It is because any change in the applied voltage will modify the electric field and exciton density simultaneously, and all of them can cause change in MEL.

In this letter, an OLED structure with the composite light emitting layer (EML) is designed to tune the MEL. The MEL is optimized by change the EML thickness and the volume ratio of $\mathrm{N}, \mathrm{N}^{\prime}$-bis(1-naphthyl)-N, $\mathrm{N}^{\prime}$-diphenyl-1, 1' -biphentl$4,4^{\prime}$-diamine (NPB) of the EML. Our results show that MEL is very sensitive and inversely related to the carrier mobility of the organic semiconductors. The relationship between the carrier mobility and the MEL is verified by the carrier hopping rate through our theoretical study of the MEL. The results confirm that MEL can contribute to diagnose the carrier mobility of an organic semiconductor.

The device structure is shown in Fig. 1(a). The fluorescent EML with a thickness of $20 \mathrm{~nm}$ composes of tris-(8hydroxyquinoline) aluminum $\left(\mathrm{Alq}_{3}\right)$ and NPB. The emitting area is approximately $4 \times 4 \mathrm{~mm}^{2}$. The 40 -nm-thick NPB and $50 \mathrm{~nm}$-thick $\mathrm{Alq}_{3}$ form the hole-transport and electrontransport layers, respectively. ITO and Al/LiF are anode and composite cathode, respectively. By applying magnetic field to the OLEDs, the brightness of the EL will be increased.

\footnotetext{
${ }^{\text {a) }}$ Author to whom correspondence should be addressed. Electronic mail: chchoy@eee.hku.hk.
}

Figure 1(b) shows the $\triangle E L / E L$ (i.e., MEL) of the OLEDs with eight different volume ratios of NPB varying from $0 \%$ to $100 \%$. At low magnetic field (i.e., $0-15 \mathrm{mT}$ ), the increment of brightness is large, while at high magnetic field ( $>20 \mathrm{mT}$ ), the brightness almost saturates. The inset of Fig. 1(b) shows that the normalized MELs of eight devices have the same trend. The result indicates that the features of MEL
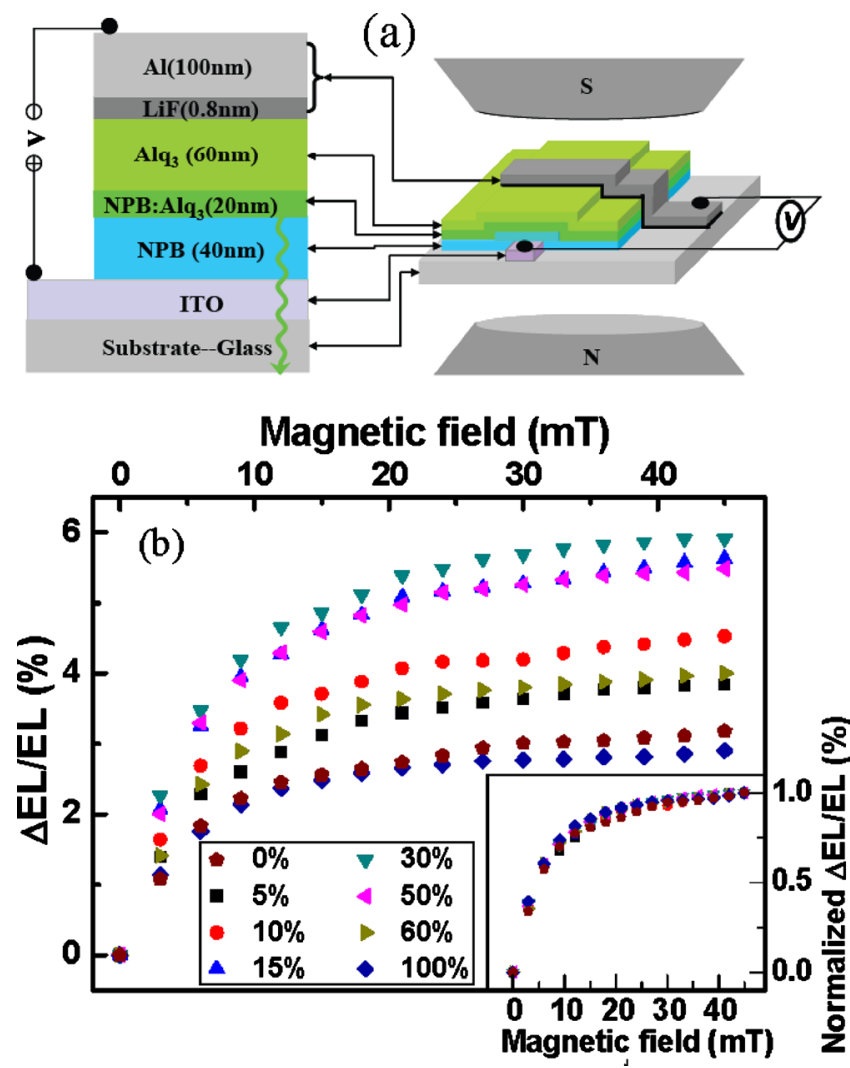

FIG. 1. (Color online) (a) A schematic view of the device structure. (b) The experimental MEL of the eight OLEDs with different NPB concentrations in the EML. All devices are driven at $500 \mu \mathrm{A}$. Inset shows the normalized MEL. 

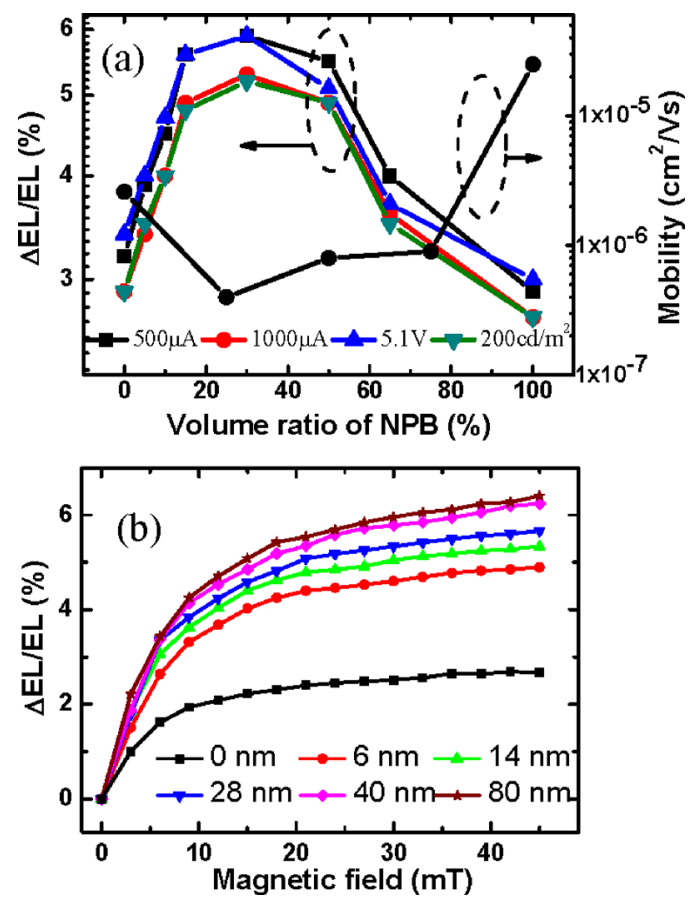

FIG. 2. (Color online) (a) MEL vs the volume ratio of NPB of the mixed EML at a fixed magnetic field of $45 \mathrm{mT}$. Four different driving modes including constant current driving of 500 and $1000 \mu \mathrm{A}$, constant voltage driving of $5.1 \mathrm{~V}$ and constant initial brightness of $200 \mathrm{~cd} / \mathrm{m}^{2}$. Black circle dot denotes the experimental electron mobility of NPB: $\mathrm{Alq}_{3}$ mixed materials taken from Ref. 15. (b) MEL of the six devices with different EML thicknesses. The OLEDs were driven at $1000 \mu \mathrm{A}$.

(i.e., $\Delta \mathrm{EL} / \mathrm{EL}$ ) with the strength of magnetic field are insensitive with the change in concentration ratio but not the true value of $\Delta \mathrm{EL} / \mathrm{EL}$.

In order to intuitively show the concentration effects (and thus study the mobility effects) of the mixed EML on the value of $\Delta \mathrm{EL} / \mathrm{EL}$, the $\Delta \mathrm{EL} / \mathrm{EL}$ of the eight devices at a given magnetic field of $45 \mathrm{mT}$ is plotted in Fig. 2(a). The devices are driven at four different modes. Take the MEL under the constant driving current of $1000 \mu \mathrm{A}$ as an example, by varying the volume ratio of NPB from $0 \%$ to $30 \%$, $\Delta \mathrm{EL} / \mathrm{EL}$ increases from $2.9 \%$ to $5.3 \%$. When the volume ratio is further increased from $30 \%$ to $100 \%, \Delta \mathrm{EL} / \mathrm{EL}$ decreases from $5.3 \%$ to $2.7 \%$. The optimized volume ratio therefore is $30 \%$. The MELs under other three driving modes including constant current $500 \mu \mathrm{A}$, constant voltage $5.1 \mathrm{~V}$, and constant initial brightness $200 \mathrm{~cd} / \mathrm{m}^{2}$ all reveal that $30 \%$ is the optimized ratio for maximizing the MEL. More importantly, the unique feature of the MEL with the concentration of the mixed EML is merely from the concentration effect and not due to the driving conditions. The unique feature can be used to diagnose the carrier mobility of the organic semiconductor as will be discussed later.

In fact, the MEL is can be further enhanced by changing the thickness of the mixed EML. Figure 2(b) shows the OLEDs with six different EML thickness including 0, 6, 14, 28,40 , and $80 \mathrm{~nm}$. The device structure is $\mathrm{ITO} / \mathrm{NPB}(50$ $-\mathrm{x} / 2 \mathrm{~nm}) / \mathrm{NPB}: \mathrm{Alq}_{3}(\mathrm{x} \mathrm{nm}) / \mathrm{Alq}_{3}(60-\mathrm{x} / 2) / \mathrm{LiF} / \mathrm{Al}$. The total thickness of the devices is constant and all volume ratio of NPB in the mixed EML is fixed at $30 \%$. The normalized $\triangle \mathrm{EL} / \mathrm{ELs}$ of these OLEDs show the similar trend as that in the inset of Fig. 2(a). Consequently, the trend of MEL with the strength of magnetic field is insensitive with the change in concentration and thickness of the EML.

Concerning the magnitude of the $\Delta \mathrm{EL} / \mathrm{EL}$, when the thickness of EML increases from 0 to $40 \mathrm{~nm}$, the magnitude increases in the whole range of the applied magnetic field. For the thickness from 40 to $80 \mathrm{~nm}$, the enhancement with thickness of blended layer is almost saturated. As shown in Fig. 2(b), at the given magnetic field of $45 \mathrm{mT}, \Delta \mathrm{EL} / \mathrm{EL}$ increases from $2.5 \%$ to $6.3 \%$ when the thickness increases from 0 to $40 \mathrm{~nm}$. However, $\Delta \mathrm{EL} / \mathrm{EL}$ only changes from $6.3 \%$ to $6.5 \%$ when the thickness is further increased from 40 to $80 \mathrm{~nm}$. Consequently, with the increase in emission region, the $\Delta \mathrm{EL} / \mathrm{EL}$ can be increased and optimized at about $40 \mathrm{~nm}$.

Interestingly, we find that MEL is very sensitive to the carrier mobility of the devices. When the electrical bias to the OLED reduces, the whole $\Delta \mathrm{EL} / \mathrm{EL}$ spectrum with the magnetic field reduces except the case of no magnetic field at which $\Delta \mathrm{EL} / \mathrm{EL}=0$ (the plot is not shown). For a given OLED, considering $V=\int_{0}^{d} E(x) d x$, where $\mathrm{V}$ is the driving voltage, $\mathrm{E}$ is the electric field and $d$ is the thickness of the device. When the voltage reduces, the electric field in the organic structure reduces. Meanwhile, through the formula of $\mu(E)=\mu(0) \exp (\gamma \sqrt{E})$, where $\mu(E)$ is the carrier mobility at an applied field of $E, \mu(0)$ is the zero-field carrier mobility and $\gamma$ is the Poole-Frenkel slope, we can see that when the electric field reduces, the carrier mobility drops but the $\Delta$ EL/EL (i.e., MEL) increases. This feature can be confirmed by our results as shown in Fig. 2(a). The results show that the $\Delta \mathrm{EL} / \mathrm{EL}$ is maximized when the volume ratio of NPB is around $30 \%$. We also plot the electron mobility of the mixed NPB: $\mathrm{Alq}_{3}$ taken from Ref. 15. Remarkably, the peak of the $\Delta \mathrm{EL} / \mathrm{EL}$ is almost at the trough of the measured mobility. ${ }^{15,16}$

In theory, we have introduced the Hubbard model $^{17}$ to describe the feature trend of the $\Delta \mathrm{EL} / \mathrm{EL}$. The Hamiltonian, including both the intermolecular transportation of electrons/ holes and the interaction between electrons/holes and nuclear spins, is described as,

$$
H=H_{1}+H_{2},
$$

where $H_{1}$ includes hopping and Coulomb interaction of carriers as

$$
\begin{aligned}
H_{1}= & -\sum_{i, j, \sigma}\left(t_{i, i^{\prime}}^{h} d_{i, \sigma}^{*} d_{i^{\prime}, \sigma}+t_{j, j^{\prime}}^{e} c_{j, \sigma}^{*} c_{j^{\prime}, \sigma}+h . c .\right) \\
& +U \sum_{i, j}\left(n_{i, \uparrow}^{h} n_{i, \downarrow}^{h}+n_{i, \uparrow}^{e} n_{i, \downarrow}^{e}\right)+V .
\end{aligned}
$$

In Eq. (2), $d_{j, \sigma}^{*}\left(d_{j, \sigma}\right)$ and $c_{j, \sigma}^{*}\left(c_{j, \sigma}\right)$ are the creation (annihilation) operators of hole and electron, respectively. The hole and electron have spin $\sigma$ which can be spin up ( $\uparrow)$ or down $(\downarrow)$ in the $i$ th and $j$ th molecule. $i^{\prime}$ and $j^{\prime}$ denote the neighbors of the $i$ th and $j$ th molecule, respectively, $t_{i, i^{\prime}}^{h}\left(t_{j, j^{\prime}}^{e}\right)$ is the hopping rate of hole between the $i$ th and $i^{\prime}$ th molecules (electron between $j$ th and $j^{\prime}$ th molecules) with a unit of microelectron volt. $U$ is the Coulomb repulsive energy between two holes or two electrons with different spins at the same molecule. $n_{i, \sigma}^{h}\left(\equiv d_{i, \sigma}^{*} d_{i^{\prime}, \sigma}\right)$ and $n_{i, \sigma}^{e}\left(\equiv c_{j, \sigma}^{*} c_{j^{\prime}, \sigma}\right)$ are the corresponding creation and annihilation multiplied operators of hole and electron, and $V$ is the attractive interaction between hole and electron at the same molecule. 


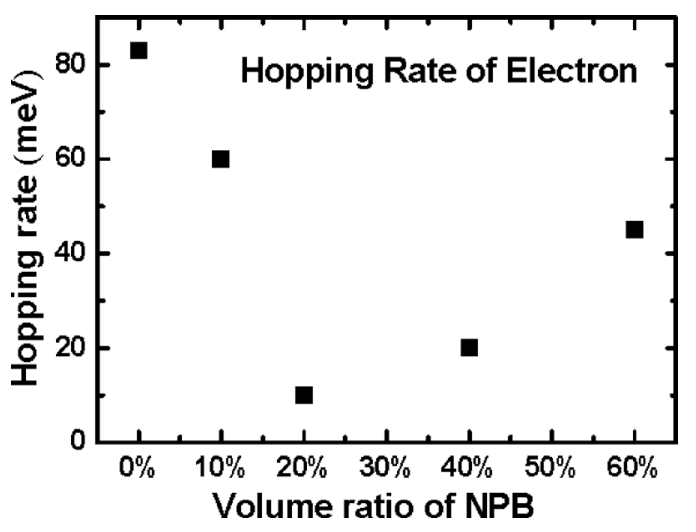

FIG. 3. The theoretical electron hopping rate of the OLEDs with NPB: $\mathrm{Alq}_{3}$ mixed EML.

$\mathrm{H}_{2}$ includes the effect of an external magnetic field and hyperfine interactions and can be expressed as

$$
H_{2}=g \mu_{B} \sum_{i, j}\left[\left(\vec{B}_{\mathrm{ext}}+\vec{B}_{\mathrm{hyp}, i}\right) \cdot \vec{S}_{i}^{h}+\left(\vec{B}_{\mathrm{ext}}+\vec{B}_{\mathrm{hyp}, j}\right) \cdot \vec{S}_{j}^{e}\right],
$$

where $g=2.0$ for organic materials, ${ }^{18} \mu_{B}$ is Bohr magnetron, $\vec{B}_{\text {ext }}$ is the external magnetic field chosen to be along the z-direction, and $\vec{B}_{\mathrm{hyp}, i(j)}$ is the effective nuclear magnetic field of the $i$ th (jth) molecule, $\vec{S}_{i}^{h}$ and $\vec{S}_{j}^{e}$ are the classical spin vectors of the hole and electron in the $i$ th and $j$ th molecules, respectively. In this work, we mainly study the relationship between the hopping rate of the carrier and MEL. Therefore, only the hopping rate is variable. Generally, hopping rates $t_{i, i^{\prime}}^{h}$ and $t_{j, j^{\prime}}^{e}$ vary from molecule to molecule due to the disorder arrangement of organic materials. Their values are typically between 1 and $100 \mu \mathrm{eV} .^{15,19}$

With the Hubbard model, the theoretical hopping rate of electron can be determined as shown in Fig. 3. It can be seen that the change in the hopping rate of electron with the volume ratio of NPB in the mixed EML is with the similar trend as that of the experimental electron mobility as shown in Fig. 2(a). The MEL is inverted related to the carrier hopping rate and mobility. Meanwhile, the maximum MEL can be obtained when the carrier mobility is at its minimum. This can provide further evidence that MEL can be used as a tool to diagnose the carrier mobility of an organic material.

In conclusion, we have presented how to use the composite EML layer compositing from NPB and $\mathrm{Alq}_{3}$ to tune and control the MEL of organic semiconductor devices. The optimized MEL is achieved when the mixed EML has NPB volume ratio of $30 \%$ and thickness of $40 \mathrm{~nm}$. Our results show that carrier mobility is a dominant factor to determine the intensity of MEL. The trend of MEL is opposite to that of the mobility of organic semiconductors. Our finding offers a way to tune MEL in OLEDs. In addition, we can use MEL as a tool to reflect the mobility of OLEDs.

This work is supported by the UGC Grant (Grant No. 400327) of the University of Hong Kong, the grants (Grant Nos. HKU712108 and HKU712010) from the Research Grant Council of the HK Special Administrative Region, China, the National Natural Science Foundation of China and Shanghai Science and Technology Commission (Grant No 08JC1402300), and the MST of China (Grant No. 2009CB929200). B. F. Ding acknowledges the support of Engineering Postdoctoral Fellowship, HKU.

${ }^{1}$ T. D. Nguyen, G. Hukic-Markosian, F. J. Wang, L. Wojcik, X. G. Li, E. Ehrenfreund, and Z. V. Vardeny, Nature Mater. 9, 345 (2010).

${ }^{2}$ P. Chen, Y. L. Lei, Q. L. Song, Y. Zhang, R. Liu, Q. M. Zhang, and Z. H. Xiong, Appl. Phys. Lett. 95, 213304 (2009).

${ }^{3}$ L. Y. Xin, C. N. Li, F. Li, S. Y. Liu, and B. Hu, Appl. Phys. Lett. 95, 123306 (2009).

${ }^{4}$ F. J. Wang, H. Bassler, and Z. V. Vardeny, Phys. Rev. Lett. 101, 236805 (2008).

${ }^{5}$ H. Odaka, Y. Okimoto, T. Yamada, H. Okamoto, M. Kawasaki, and Y. Tokura, Appl. Phys. Lett. 88, 123501 (2006).

${ }^{6}$ M. Wohlgenannt and Z. V. Vardeny, J. Phys.: Condens. Matter 15, R83 (2003).

${ }^{7}$ V. Dediu, L. E. Hueso, I. Bergenti, and C. Taliani, Nature Mater. 8, 707 (2009).

${ }^{8}$ Z. H. Xiong, D. Wu, Z. V. Vardeny, and J. Shi, Nature (London) 427, 821 (2004).

${ }^{9}$ B. F. Ding, Y. Q. Zhan, Z. Y. Sun, X. M. Ding, X. Y. Hou, Y. Z. Wu, I. Bergenti, and V. Dediu, Appl. Phys. Lett. 93, 183307 (2008).

${ }^{10}$ G. Li, J. Shinar, and G. E. Jabbour, Phys. Rev. B 71, 235211 (2005).

${ }^{11}$ Z. H. Xu, B. Hu, and J. Howe, J. Appl. Phys. 103, 043909 (2008).

${ }^{12}$ B. Hu and $\mathrm{Y}$. Wu, Nature Mater. 6, 985 (2007).

${ }^{13}$ K. Maeda, K. B. Henbest, F. Cintolesi, I. Kuprov, C. T. Rodgers, P. A. Liddell, Devens Gust, C. R. Timmel, and P. J. Hore, Nature (London) 453, 387 (2008).

${ }^{14}$ P. Desai, P. Shakya, T. Kreouzis, W. P. Gillin, N. A. Morley, and M. R. J. Gibbs, Phys. Rev. B 75, 094423 (2007).

${ }^{15}$ S. W. Liu, J. H. Lee, C. C. Lee, C. T. Chen, and J. K. Wang, Appl. Phys. Lett. 91, 142106 (2007).

${ }^{16}$ C. H. Hsiao, Y. H. Chen, C. T. Lin, C. C. Hsia, and J. H. Lee, Appl. Phys. Lett. 89, 163511 (2006)

${ }^{17}$ A. Auerbach, Interacting Electrons and Quantum Magnetism (Springer, New York, 1998).

${ }^{18}$ D. R. McCamey, H. A. Seipel, S.-Y. Paik, M. J. Walter, N. J. Borys, J. M. Lupton, and C. Boehme, Nature Mater. 7, 723 (2008).

${ }^{19}$ A. J. Drew, F. L. Pratt, J. Hoppler, L. Schulz, V. Malik-Kumar, N. A. Morley, P. Desai, P. Shakya, T. Kreouzis, W. P. Gillin, K. W. Kim, A. Dubroka, and R. Scheuermann, Phys. Rev. Lett. 100, 116601 (2008). 